\title{
DISORDERS OF RHYTHM APPEARING AFTER IMPLANTATION OF SYNCHRONIZED PACEMAKERS
}

\author{
BY \\ AGUSTIN CASTELLANOS JR. AND LOUIS LEMBERG \\ From the Section of Cardiology, Department of Medicine, University of Miami School of Medicine, and the Department \\ of Electrophysiology, Jackson Memorial Hospital, Miami, Florida, U.S.A. \\ Received January 20, 1964
}

Analysis of clinical arrhythmias has resulted in a closer understanding of the basic electrophysiology of the human heart (Scherf and Schott, 1953; Katz and Pick, 1956; Scherf and Cohen, 1964).

The experimental animal has not been the only source of knowledge in the broad field of disorders of impulse formation and impulse conduction (Linenthal and Zoll, 1962; Katz and Pick, 1963). The recent introduction of implantable cardiac pacemakers for the treatment of complete and high-grade atrio-ventricular (A-V) block has made it possible to increase our knowledge of those arrhythmias resulting from the simultaneous activities of several spontaneous pacemakers, by comparing them with those due to interaction of a spontaneous with an artificial pacemaker (Soloff, 1962; Burchell, 1963). Of the several types available we have been interested mainly in the synchronized pacemaker of Nathan et al. (1963), since the cardiac rhythm induced by this instrument approximates to a normal sinus rhythm. The artificial ventricular pacemaker is triggered by an amplified atrial action potential which is then fed into the ventricular pacemaker with a delay simulating that of the A-V interval in normal sinus rhythm. As a safety precaution a standby device is also incorporated, activating the ventricular pacemaker after an adjustable interval should activation from the atria fail. Prevention of a rapid ventricular response to atrial tachycardias, flutter, or fibrillation is similarly controlled through a refractory delay in the pacer of a little over 0.5 second. This allows the ventricles to respond at a rate not exceeding 110 a minute. At atrial rates of 111 to 220 a $2: 1$ block is induced, and above 221 the ventricular response is in the order of $3: 1$. Nathan et al. developed this type of artificial pacemaker on the assumption that the efficacy of a pacemaker is greater the more it resembles the normal cardiac mechanism. Thus, in instances where normal A-V conduction is impaired, the introduction of a system providing synchronized conduction with physiological A-V delay results in enhanced cardiac output when compared with unsynchronized ventricular pacing (Braunwald and Frahm, 1961). A detailed description of this synchronized pacemaker has been fully reported elsewhere and will, therefore, not be considered in this communication (Nathan et al., 1963). This report is mainly concerned with the description of arrhythmias, heretofore not described in man, which have been observed following implantation of this pacemaker. In addition, the resemblance between the behaviour of man-induced and spontaneous pacemakers will be stressed.

\section{ISOLATED PACEMAKer ESCAPES}

This is the simplest arrhythmia that can occur in patients in which the device has been implanted. An escape is considered to be a safety mechanism which preserves the function of the heart whenever the primary pacemaker does not initiate impulses, or when these for one reason or another fail to reach the A-V node (Scherf and Schott, 1953). In such instances either an A-V junctional or an 
idioventricular escape can occur. The similarity between artificial pacemaker escapes and the naturally occurring phenomenon can be seen in Fig. 1. The records were obtained from a 61-yearold patient who previously had complete A-V block and Adams-Stokes seizures. Every atrial impulse (A) is delivered to the pacemaker $(\mathrm{P})^{*}$, followed by a delay equivalent to an A-P conduction time of $0 \cdot 18$ second. The pacemaker then depolarizes the ventricles $(\mathrm{V})$. The sino-atrial rate is 88 a minute (A-A interval 0.68 second). A blocked atrial extrasystole (A.Ex.), occurring 0.48 second after the fourth A wave, discharges the sinus node. After an interval of 1.07 seconds during which there is no ventricular contraction ( $\mathrm{P}-\mathrm{P}$ of $1.07=$ rate of 56 per minute), the artificial pacemaker escapes and stimulates the ventricles. The escape interval was preset in the pacer as a precaution so that a spontaneous discharge occurs whenever there is a pause of close to 1.07 seconds.

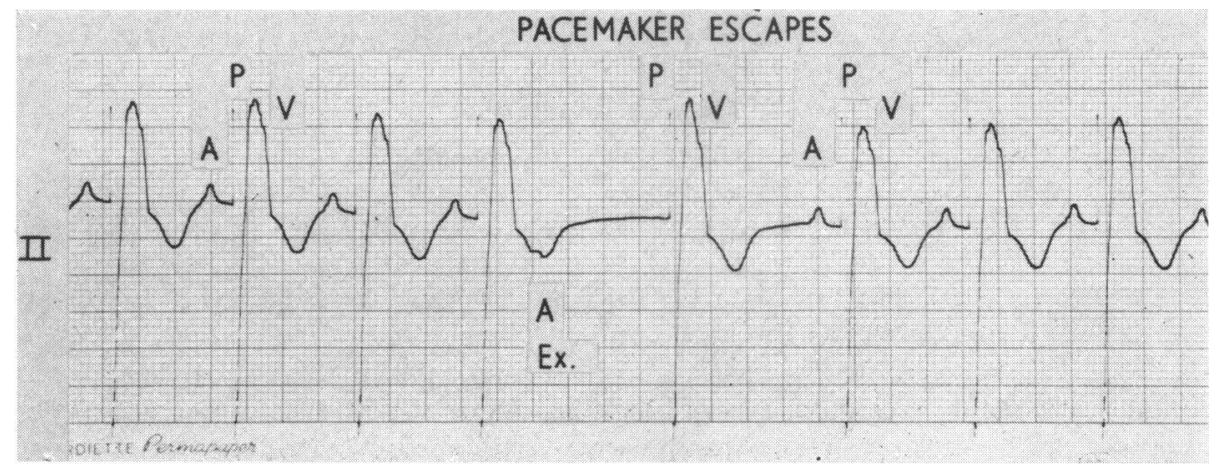

FIG. 1.-Pacemaker "escape" occurring after a blocked atrial extrasystole (A.Ex.). In this, and in all illustrations, A represents atrial activity; P, the stimulus artefact produced by the artificial pacemaker; and V, ventricular depolarization. All figures are fully described in text.

The record also illustrates that pacemaker escapes, as well as its A-V nodal and idioventricular counterparts, occur after a larger interval than that occurring between sinus beats. In addition, a succession of escapes (A-V dissociation) did not occur in this patient because the following sinoatrial contraction (fifth) activated the pacemaker 0.96 second after the escape (P-P of $0.96=62$ a minute).

\section{Dissociation Between Atria and Pacemaker (INTERference-Dissociation)}

An arrhythmia typical of interference dissociation was noted in one case with a successfully implanted synchronized pacer. The pacemaker escaped from atrial control for periods when the atrial rate slowed and (if) the pacer rate was set at relatively high rates. Interference dissociation is a disorder of rhythm in which the ventricles (usually under the command of the A-V node) beat independently and faster than the atria. This occurs with unidirectional V-A block which prevents retrograde atrial depolarization (Mobitz, 1923). The supraventricular impulse may or may not capture the ventricles depending on the relation between the rates of the supraventricular and ventricular rhythms, the A-V conduction time of supraventicular impulses, and the length of the refractory period of the junctional tissues and ventricles (Scherf and Schott, 1953). A ventricular capture (Katz and Pick, 1956) or "interference" beat (Scherf and Schott, 1953) is characterized usually by a shortening of the corresponding $\mathrm{R}-\mathrm{R}$ cycle.

Figure 2, obtained from a 63-year-old patient with chronic complete A-V block, shows dissociation between the sino-atrial node (A) and an artificial pacemaker (P). The rate of the latter is 86 a

* Note that the abbreviation $(\mathrm{P})$ is used throughout this communication only in reference to the pacemaker stimulus artefact and not in its usual fashion (see legend to Fig. 1). 
minute (P-P intervals of 0.70 second), and is more rapid than the supraventricular centre (75 a minute). The fourth atrial contraction captures the pacemaker as evidenced by the shortened P-P interval of 0.65 second. The corresponding A-P conduction time is 0.18 second. These features satisfy the necessary requirements of interference dissociation.

It is not unusual for the ventricular rate to be more rapid than the atrial rate when the heart is electrically paced, but ventricular captures are diagnostic features of interference dissociation. The unusual occurrence in this case is that the ventricles are captured via the artificial pacemaker. The supraventricular impulse "captures" the electronic pacemaker which in turn stimulates and depolarizes the ventricles. This sequence of events simulates interference dissociation, and since this is artificially produced the term atrio-pacemaker (A-P) interference dissociation is introduced.

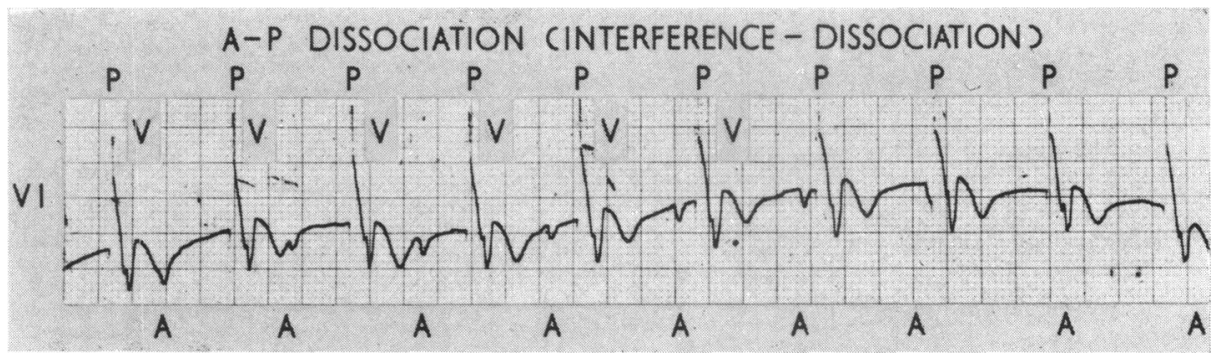

FIG. 2.-Dissociation between atria (A) and artificial pacemaker (P) (interference-dissociation). Note that the fourth sino-atrial impulse "captures" the artificial pacemaker.

\section{Dissociation Between Atria and Pacemaker in Presence of High Degree A-P Block}

In addition to typical interference-dissociation (Mobitz, 1923), in which the ventricles beat faster than the atria, there are instances in which the rates of both pacemakers are more or less the same. The resultant arrhythmia is referred to as an isorhythmic A-V dissociation (Segers, Enderle, and Pirart, 1953; Schubart, Marriott, and Gorten, 1958). At times the relation between atrial and ventricular contractions can be constant (spontaneous synchronization) (Segers, 1946): in these cases the correct interpretation can be made only if long records are available, or after carotid sinus pressure, or the Valsalva manœuvre. Neurogenic stimuli have dissimilar effects upon the two centres of impulse formation (Azan, Castellanos, and Calvino, 1958).

Another interesting arrhythmia described by Dressler in 1929 is dissociation in the presence of apparent 2:1 A-V block. Dissociation is present even though the atrial rate is faster than the ventricular rate. Complete A-V block does not exist, since at times supraventricular impulses are able to capture the ventricles. An indispensable condition of this disorder of rhythm is the fact that even though the ventricular rate is slower than the atrial rate it exceeds the conducted atrial rate. Simply stated, the ventricular rate is greater than half the atrial rate.

Figure 3 was obtained from an 82-year-old man in whom the pacemaker was implanted for the treatment of chronic complete A-V block and Adams-Stokes seizures of recent onset. Analysis of the upper strip taken one day after operation revealed that the atria controlled the pacemaker, which in turn depolarized the ventricles.

The lower strip illustrates the usual finding seen when an artificial pacer operates in the presence of normal orthograde conduction of some beats. The resultant arrhythmia has been considered akin to spontaneous parasystole; hence the name, iatrogenic parasystole (Soloff, 1962). However, close inspection of the strip reveals that the basic difference between this and similar published examples lies in the fact that the atria do not capture the ventricles directly. For instance, the first supraventricular beat discharges the pacer with an A-P interval of $0 \cdot 20$ second. The second sinoatrial impulse does not activate the pacemaker, because the refractory period of the pacemaker was set at 0.60 second. Thus, when the second atrial impulse appeared within the refractory interval, 


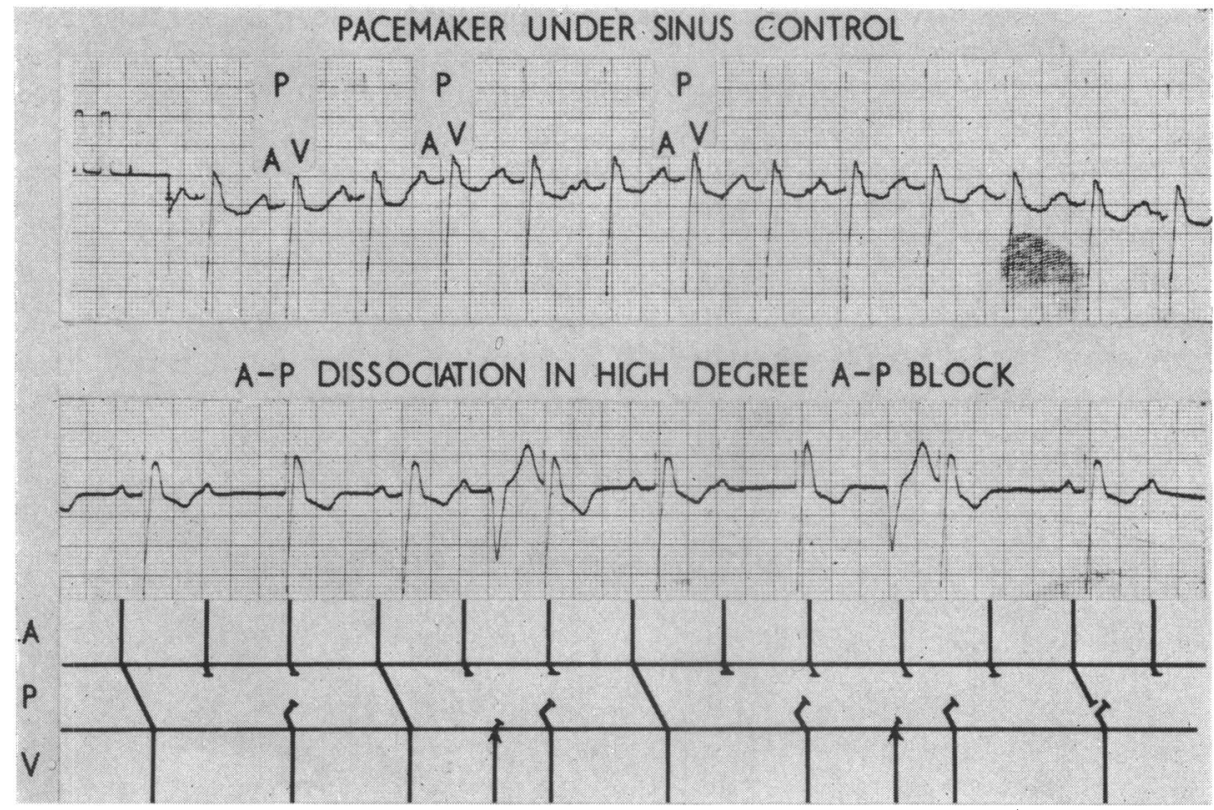

FIG. 3.-In the upper strip there is synchronized activity of atria, pacemaker, and ventricles. The lower strip shows pacemaker "escapes", as well as atrio-pacemaker dissociation in the presence of 2-1 A-P "block". The conventions are as follows: A-P represents the propagation of impulses through the pacemaker $(P)$, which conducts the impulses from the atria $(A)$ to the ventricles $(V)$. Oblique lines indicate speed of transmission, and the short lines at right angles from the former indicate stoppage of impulses. Note that the artificial pacemaker permits only orthograde conduction; consequently its spontaneous discharges, as well as ventricular extrasystoles, are not conducted retrogradely to the atria through the instrument.

it was "blocked" (non-conducted). In this sense the refractory period of the electronic pacemaker behaves in a fashion similar to that of the junctional tissues of the heart.

Further analysis of the tracing reveals that the second pacemaker discharge is spontaneous and not conditioned to the preceding supraventricular impulse. This pacer discharge occurs at a specific instance because of the pre-set escape interval of one second which is a built-in safety feature. The pacer escape beat then interferes with the third A wave. Finally, the fourth A wave again stimulates the pacemaker.

A similar sequence of events can be observed after the seventh A wave: however this is followed by a succession of escapes (A-P dissociation) at a rate of 60 a minute (P-P intervals of $1.00 \mathrm{sec}$.). Another feature of interest in this case is the fact that the escapes are not influenced by the premature ventricular contractions. The record, which is the artificial counterpart of $\mathrm{A}-\mathrm{V}$ dissociation in $2: 1$ A-V block, presents the following analogy with the latter: the iatrogenic pacemaker is slower than the sinus node (60 a minute), but faster than half the atrial rate (51 a minute). It is interesting to note that by chance occurrence the rate of the atrial pacemaker, in which A-V dissociation was artificially produced by an internal pacemaker in a patient with $2: 1 \mathrm{~A}-\mathrm{V}$ block, was similar to that in our case (Dressler and Jonas, 1962).

\section{Evidence of a DuAl A-V Conducting System}

The possibility of the presence of dual pathways for A-V and V-A conduction in man as well as in the experimental animal has been previously emphasized. However, there is disagreement as to whether the dual pathways imply a normal A-V junction with various refractory periods or the 


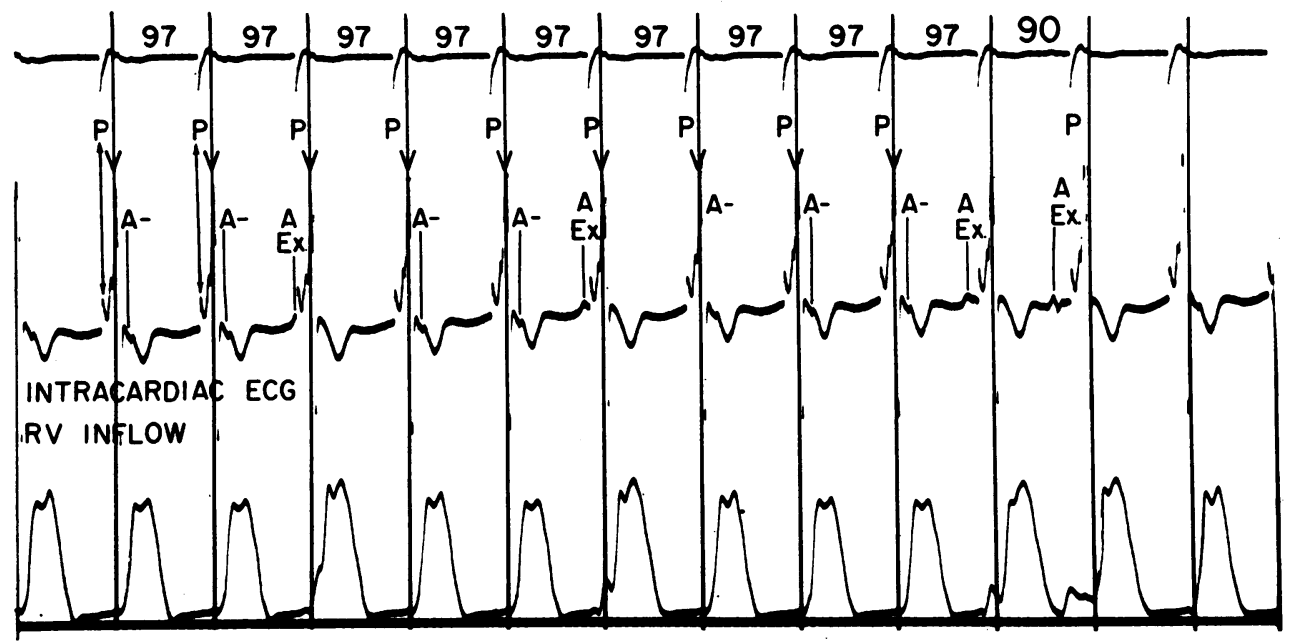

Fig. 4.-Forward conduction through the artificial pacemaker and retrograde conduction through the natural A-V junction. A-represents retrograde depolarization of the atria, by the ventricular contractions; A-Ex., is used to designate spontaneous supraventricular impulses, probably ectopic atrial in origin. The upper recording is a standard lead in which the stimulus artefact is very prominent; the middle tracing is an intracardiac electrocardiogram recorded at the inflow tract of the right ventricle. The right ventricular pressure curve can be seen at the bottom.

simultaneous presence of both normal and ectopic atrio-ventricular communications. In the last decade there has been added interest in the dual A-V transmission system especially since the description by physiologists of the "echo" mechanism (Moe, Preston, and Burlington, 1956; Rosenblueth and Rubio, 1958). This is the experimental counterpart of the well-known electrocardiographic phenomenon of return extrasystoles (Scherf, 1941) or reciprocal rhythm (Katz, 1946).

Some authors (James, 1963) minimize the importance of ectopic atrio-ventricular connexions. Others believe that these connexions, confirmed anatomically (Lev, Sodi-Pallares, and Friedland, 1963), can function under certain circumstances and are important in the mechanism of the preexcitation (W-P-W) syndrome (Scherf and Schott, 1953; Katz and Pick, 1956).

An example of this phenomenon can be seen in Fig. 4. The record is that of a 59-year-old man in whom the synchronized pacemaker was implanted because of advanced A-V block and AdamsStokes seizures. Several days after operation, the sinus node slowed to a rate below that of the mechanical pacer resulting in an automatic ventricular rate of 61 a minute (pacemaker interval of 0.97 second). Retrograde conduction to the atria, evidenced by the inverted $P$ wave (labelled A), can be seen in the descending portion of many $T$ waves. Since the intracardiac electrode was at the inflow tract of the right ventricle the negative $P$ wave suggests retrograde activation of the atria.

This phenomenon is not so rare in atrio-ventricular block (Winternitz and Langendorf, 1944) and has also been described in patients in whom artificial pacemakers have been implanted (Linenthal et al., 1962; Adams, 1963; Burchell, 1963). Though the possibility of isorhythmic A-V dissociation could be considered, examination of long strips showing such a fixed ventriculo-atrial relation makes this possibility unlikely (Azan et al., 1958). Further analysis shows the presence of scattered premature atrial contractions (A.Ex.). The first three premature beats reach the pacemaker after its discharge. The respective QRS complexes are not followed by retrograde conduction to the atria because of the refractory period of the latter. The last premature atrial contraction occurs at a shorter P-A Ex. interval than the succeeding three $(0.74 \mathrm{sec} .<0.94,0.93$, or 0.92 second $)$ and thus captures the iatrogenic pacemaker before the latter discharges spontaneously. The P-P interval of the capture is shorter than that of the sinus rate $(0.90$ and 0.97 second). In addition, the record reveals that the stimulus has two A-V pathways: the V-A conducting system in its journey 
towards the atria; and the anomalous artificial connexion to the ventricles. A reciprocating rhythm did not occur because of the length of the refractory period of the atria and pacemaker, as well as the absence of orthograde A-V conduction.

The assumptions expressed above suggest that double connexions between atria and ventricles can operate side by side regardless of the fact that one of these is artificial. If the analogy is valid, one is led to hypothesize that the congenital accessory communications found in the human heart can function in a similar way. It is evident that this assumption in no way denies the existence of a functional longitudinal dissociation of the A-V conduction system (Scherf, 1941) or of two groups of fibres with different degrees of refractoriness located within the normal A-V junctional tissues (Moe et al., 1956; Rosenblueth and Rubio, 1958; Hoffman et al., 1963).

\section{NODAL-PACEMAKER AND VeNTRICULO-PACEMAKER RHYTHMS}

An interesting phenomenon occurred in a 62-year-old patient with high degree A-V block and Adams-Stokes seizures due to intermittent ventricular stand-still. The upper strip in Fig. 5 is a control tracing taken from this patient before operation. The last ventricular cycle, which is shorter than the preceding one $(0.88 \mathrm{sec} .<1.06 \mathrm{sec}$. $)$, represents a ventricular capture. The $P-R$ interval of 0.55 was slightly longer than those observed during brief periods of $1: 1 \mathrm{~A}-\mathrm{V}$ conduction $(0.45$ second in strips not reproduced). The identical morphology of both conducted and non-conducted beats indicates that the A-V node is the probable site of impulse formation whenever the supraventricular impulses fail to reach the ventricles. The widened and bizarre QRS complexes are attributed to intraventricular (bundle-branch) block. Pacemaker function was normal the first few

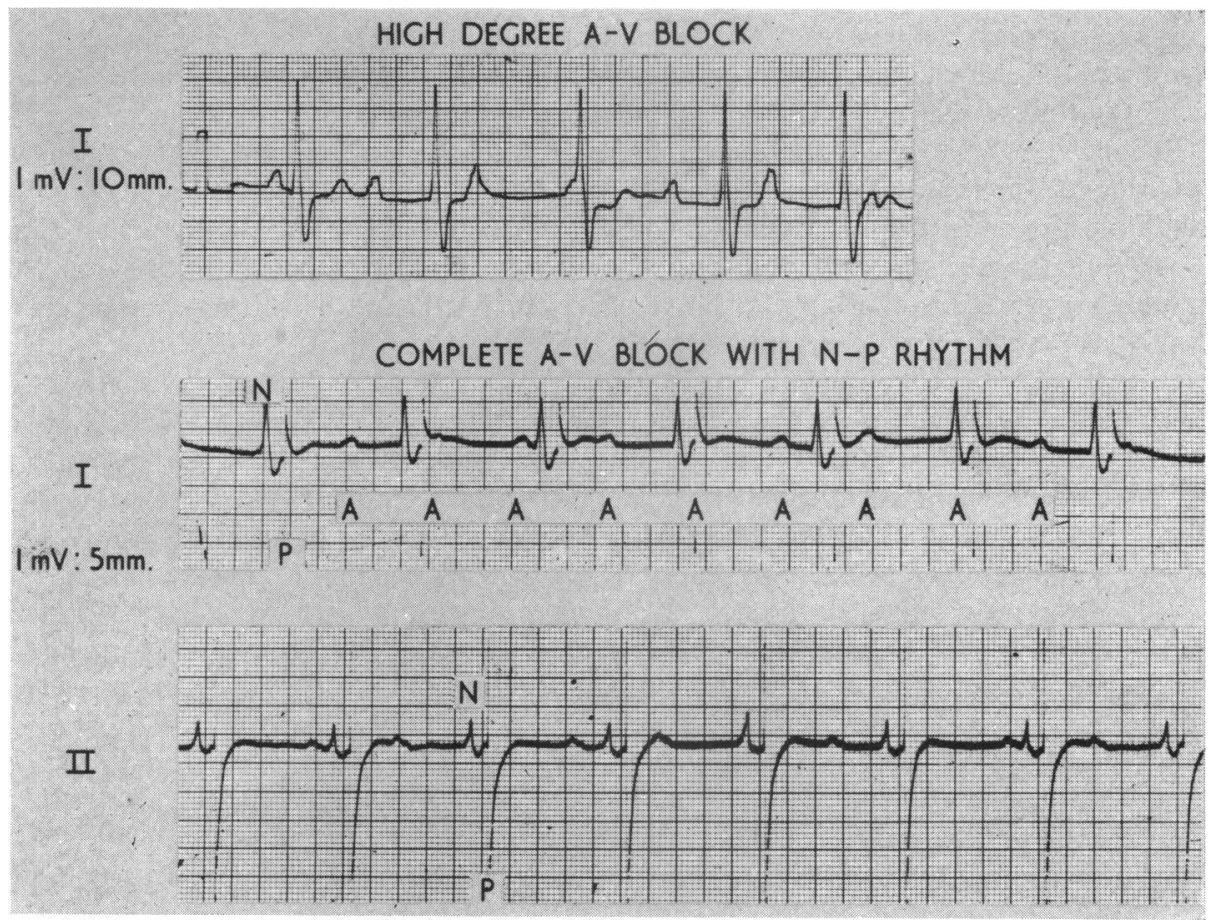

Fig. 5.-High degree A-V block with occasional ventricular captures can be seen in the upper strip. The middle and lower strips, obtained after implantation of the pacemaker, show apparently complete A-V block (note decrease in standardization). There is a fixed relation between the spontaneous A-V nodal contractions $(N)$ and the stimulus artefact $(P)$, indicating that the latter are probably dependent on the former. 


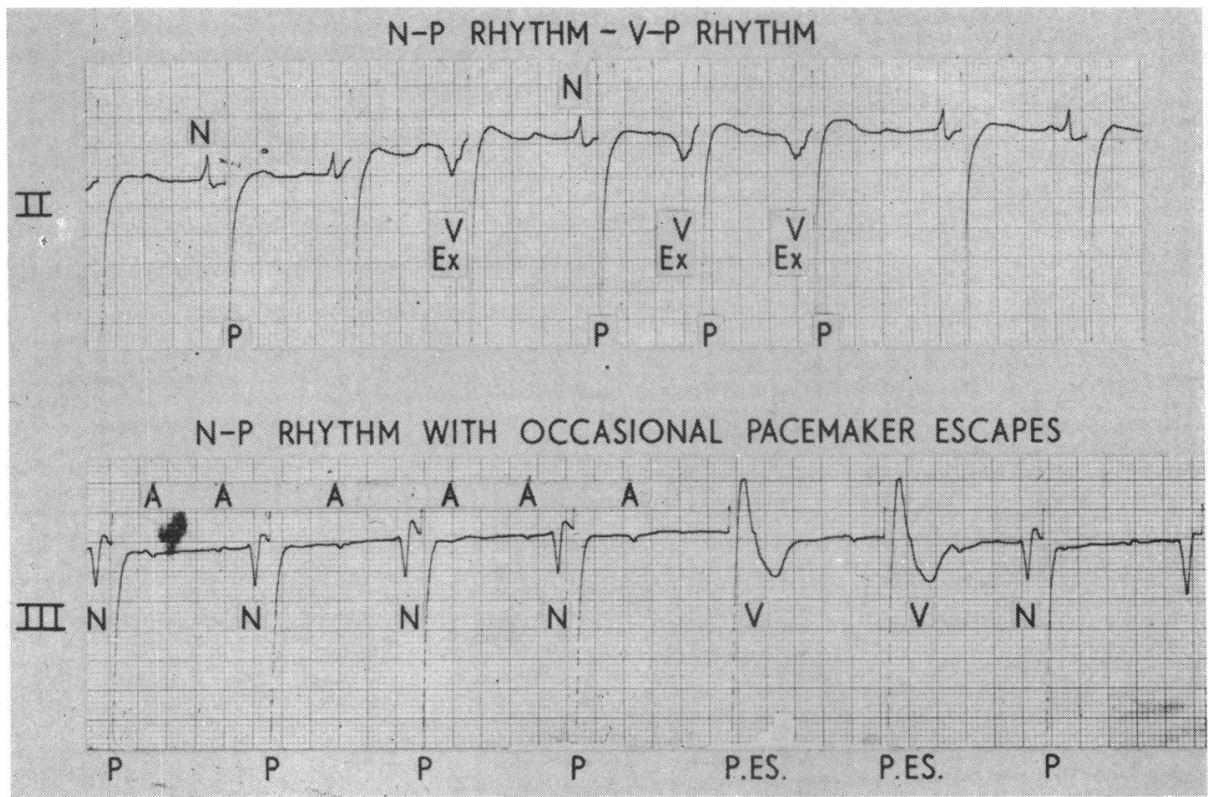

FIG. 6.-Apparently complete A-V block with spontaneous atrio-ventricular nodal rhythm (N). Observe that in the upper strip the artificial pacemaker is responding to both nodal $(\mathrm{N})$ and ectopic ventricular (V.Ex.) impulses. These bizarre, heretofore undescribed, rhythms are introduced as N-P and V-P rhythms, respectively. The lower strip shows pacemaker escapes (P.Es.) after transient depression of impulse formation in the $A-V$ node. The corresponding ventricular responses have a different contour from the previous ones.

days after operation. On the fifth post-operative day, the arrhythmia shown in the middle and lower strips of Fig. 5 was recorded: there is complete A-V block with the atrial and ventricular rates of 100 and 60 a minute, respectively. The spontaneous pacemaker responsible for ventricular activation was thought to be located in the A-V node because of its resemblance with that present before operation (note half standardization in 2nd and 3rd strips). A remarkable finding in this patient was the fixed relation between A-V nodal and iatrogenic pacemakers. Their rates were identical; yet this does not exclude isorhythmic nodal-artificial pacemaker (N-P) dissociation. In these instances a fixed N-P relation can be expected, at least for short periods. Yet no definite conclusions could be drawn from this short strip.

In Fig. 6, an arrhythmia similar to the one in Fig. 5 is seen in the first two cycles. The third QRS complex is premature, and has a different morphology than the nodal beats. This, and other beats of similar contour, are probably ventricular extrasystoles (V.Ex.). Remarkably, all premature ectopic contractions control the timing of the pacemaker artefacts, thus indicating that the latter are dependent on (and thus related to) the ventricular extrasystoles. In retrospect, it may be assumed that the artificial pacer was also triggered by the A-V nodal impulses in Fig. 5. The lower strip shows a nodal-pacemaker rhythm independent of the faster atrial impulses. The A-V nodal intervals are slightly irregular $(1 \cdot 10,1.04$, and 1.08 seconds respectively) due to an arrhythmic function of the A-V centre. It is important to note that the artificial pacemaker intervals change accordingly, indicating their dependence on the human pacemaker. When the latter is inhibited for a longer than usual period ( 1.20 seconds) the former escapes (P.Es.): the escape representing a compensatory mechanism that prevents ventricular standstill. Note that this and the next spontaneous artificial discharges are followed by QRS complexes that are different in shape from the A-V nodal and idioventricular ones. They are thought to arise in response to pacemaker stimulus at the site of electrode implantation. This tracing is significant in that there is a rare instance where the human 
nodal pacemaker governs the artificial pacemaker instead of the reverse and expected sequence. Special x-ray studies revealed the usual locations of the lead wires to the left atrium and left ventricle, thus eliminating the possibility of reversal of lead attachments during operation. The weird events occurring in this case were considered to be the result of pacemaker malfunction.

\section{SUMMARY}

Several heretofore undescribed arrhythmias that appeared after implantation of synchronized pacemakers in man have been described. The nomenclature that best fits these iatrogenic disorders of rhythm are: pacemaker escapes (from sinus control); dissociation between atria and pacemaker (A-P dissociation) with pacemaker captures (by the sinus node); the firing of the iatrogenic pacemaker by spontaneous A-V nodal beats and ectopic ventricular contractions (N-P and V-P rhythms, respectively). In one patient there was evidence in favour of a dual A-V conducting system: the normal junction used exclusively by the impulses propagating towards the atria; and the artificial communication which permitted transmission from atria to ventricles only. Finally, a rare phenomenon was presented where the implanted synchronized electronic pacemaker was paced by the human ventricle even though lead attachments in the heart were correct (an example of pacemaker malfunction).

\section{REFERENCES}

Adams, C. W. (1963). Retrograde atrial conduction with complete heart block following implantation of an internal ventricular pacemaker. Dis. Chest, $43,544$.

Azan, Cano L., Castellanos, A., Jr., and Calvino, J. M. (1958). Mecanismos de la disociacion con interferencia. Rev. cuba. Cardiol., 19, 163.

Braunwald, E., and Frahm, C. J. (1961). Studies on Starling's law of the heart. IV. Observations on the hemodynamical functions of the left atrium in man. Circulation, 24, 633.

Burchell, H. B. (1963). Analogy of electronic pacemaker and ventricular parasystole with observations on refractory period, supernormal phase and synchronization. Circulation, 27, 878.

Dressler, W. (1929). Dissoziationen und Interferenzen bei partiellem Herzblock. Z. klin. Med., 111, 23.

- and Jonas, S. (1962). Experimental production of A-V dissociation with ventricular captures by use of an internal pacemaker in patients with 2:1 A-V block. Amer. J. Cardiol., 10, 547.

Hoffman, B. F., Moore, E. N., Stuckey, J. H., and Cranefield, P. F. (1963). Functional properties of the atrioventricular conduction system. Circulat. Res., 13, 308.

James, T. N. (1963). Anatomy of the conducting system of the heart. Heart Bull., 12, 21.

Katz, A. M., and Pick, A. (1963). The transseptal conduction time in the human heart. An evaluation of fusion beats in ventricular parasystole. Circulation, 27, 1061.

Katz, L. N. (1946). Electrocardiography, 2nd ed. Lea and Febiger, Philadelphia.

, and Pick, A. (1956). Clinical Electrocardidgraphy, Part I. The Arrhythmias. Lea and Febiger, Philadelphia.

Lev, M., Sodi-Pallares, D., and Friedland, C. (1963). A histopathologic study of the atrioventricular communications in a case of Wolff-Parkinson-White syndrome with incomplete left bundle-branch block. Amer. Heart J., 66, 399.

Linenthal, A. J., and Zoll, P. M. (1962). Quantitative studies of ventricular refractory and supernormal periods in man. Trans. Ass. Amer. Phycns, 75, 285.

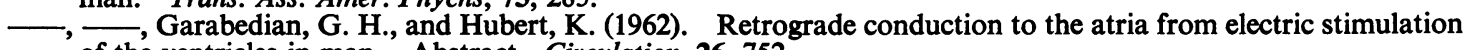
of the ventricles in man. Abstract. Circulation, 26, 752.

Mobitz, W. (1923). Zur frage der atrioventrikulären Automatie. Die Interferenzdissoziation. Dtsch. Arch. klin. Med., 141, 257.

Moe, G. K., Preston, J. B., and Burlington, H. (1956). Physiologic evidence for a dual A-V transmission system. Circulat. Res., 4, 357.

Nathan, D. A., Center, S., Wu, C. Y., and Keller, W. (1963). An implantable synchronous pacemaker for the long term correction of complete heart block. Amer. J. Cardiol., 11, 362.

Rosenblueth, A., and Rubio, R. (1958). Ventricular "echoes." Amer. J. Physiol., 195, 53.

Scherf, D. (1941). An experimental study of reciprocating rhythm. Arch. intern. Med., $67,372$. York.

- and Schott, A. (1953). Extrasystoles and Allied Arrhythmias. Heinemann, London.

Schubart, A. F., Marriott, H. J. L., and Gorten, R. J. (1958). Isorhythmic dissociation; atrioventricular dissociation with synchronization. Amer.J. Med., 24, 209.

Segers, M. (1946). Les phénomènes de synchronisation au niveau du coeur. Arch. int. Physiol., $54,87$.

, Enderle, J., and Pirart, J. (1953). Un cas de dissociation isorythmique. Acta cardiol. (Brux.), 8, 417.

Soloff, L. A. (1962). Iatrogenic parasystole and interpolated premature ventricular beats. Amer. Heart J., 63, 563.

Winternitz, M., and Langendorf, R. (1944). Auriculoventricular block with ventriculoauricular response. Report of six cases and critical review of the literature. Amer. Heart J., 27, 301. 